\title{
Acurácia da ultrassonografia com preparo intestinal no diagnóstico da endometriose profunda
}

\author{
Accuracy of ultrasonography with intestinal preparation in diagnosis of deep \\ endometriosis
}

Fernanda Nogueira Barreto ${ }^{1}$, Ivan Abreu Figueiredo ${ }^{1 *}$

\begin{abstract}
Resumo: A endometriose consiste na presença de tecido endometrial fora da cavidade uterina das mulheres em fase reprodutiva, podendo atingir de 10 a 15\% dessa população. É uma doença crônica progressiva e tem caráter recidivante. As pacientes normalmente apresentam dor pélvica e infertilidade. O diagnóstico da endometriose representa um desafio para os ginecologistas, no entanto, a decisão sobre o tipo de tratamento a ser implantado depende de uma boa anamnese, exame físico, exames laboratoriais e de imagem. Nos últimos anos, ocorreu um avanço significativo evidenciando-se a ultrassonografia transvaginal (USTV) com alcance da sua sensibilidade quanto ao diagnóstico clínico. Pesquisas apontam que este exame apresenta sensibilidade de $98 \%$ para identificação de endometriose, acometendo o retossigmoide, e de $95 \%$ para endometriose profunda na região retrocervical, com especificidade de 100 e $98 \%$ para essas duas localizações, respectivamente. A USTV demonstra ter um bom desempenho tanto para o diagnóstico dos endometriomas assim como nas lesões ovarianas iniciais da doença. Apresenta uma qualidade na resolução da estratificação das camadas da parede intestinal. Portanto, a identificação de achados ultrassonográficos associados favorece aumento na acurácia diagnóstica.
\end{abstract}

Palavras-chaves: Endometriose profunda. Diagnóstico. Acurácia. Ultrassonografia.

Abstract: Endometriosis consists of the presence of endometrial tissue outside the uterine cavity of women in the reproductive phase, which can reach 10 to $15 \%$ of this population. It is a chronic progressive disease and has recurrent character. Patients usually present with pelvic pain and infertility. The diagnosis of endometriosis poses a challenge for gynecologists, however, the decision on the type of treatment to be implanted depends on a good anamnesis, physical examination, laboratory and imaging tests. In recent years, there has been a significant advance in transvaginal ultrasonography (USTV), with sensitivity to clinical diagnosis. Research has shown that this test has a sensitivity of $98 \%$ for the identification of endometriosis, affecting retossigmoid, and $95 \%$ for deep endometriosis in the retrocervical region, with specificity of 100 and $98 \%$ for these two sites, respectively. USTV demonstrates good performance both for the diagnosis of endometriomas as well as for early ovarian lesions of the disease. It presents a quality in the stratification resolution of the layers of the intestinal wall. Therefore, the identification of associated ultrasonographic findings favors an increase in diagnostic accuracy.

Key words: Deep endometriosis. Diagnosis. Accuracy. Ultrasonography.

\footnotetext{
${ }^{1}$ Universidade Ceuma, São Luís, MA, Brasil.

* Autor correspondente - Rua Josué Montello nำ, Renascença II - São Luís-MA. CEP 65.075-

120. Telefone: +55 (98) 98868-5248. E-mail: ivanfig@terra.com.br.
} 


\section{Introdução}

Em 1960 Rokitansky considerou e definiu a endometriose pela presença de tecido endometrial fora da cavidade uterina durante a fase reprodutiva da mulher, afetando-as em torno de 10 a $15 \%$. A endometriose se expressa de forma questionável quando se refere ao seu diagnóstico. Dessa forma torna-se uma das principais patologias que incentivam publicações científicas na área da ginecologia, devido ao aumento da incidência e pelas incertezas que cercam sua precisão diagnóstica. A doença é muito comum e acomete cerca de uma paciente a cada dez, que geralmente apresentam dor pélvica e infertilidade (em até $70 \%$ dos casos) ${ }^{1}$. É uma patologia crônica progressiva e tem caráter recidivante. Quando definido o quadro, é necessário realizar um planejamento a longo prazo, preferencialmente desde a adolescência até a maturidade ${ }^{2}$.

A endometriose se apresenta na faixa etária entre 25 e 29 anos, sendo rara em pré-púberes e no climatério. Percebese a incidência elevada entre mulheres com ciclo menstrual de duração igual ou menor que 27 dias. No entanto, da mesma maneira, quando estas apresentam o tempo de sangramento é maior ou igual a sete dias e ainda acompanhada de spotting pré-menstrual ${ }^{3}$.

Além da manifestação de infertilidade por endometriose, outras queixas frequentes são dismenorréia e alterações intestinais - $84,2 \%$ e $54,4 \%$, respectivamente, sendo estas últimas associadas à diarreia cíclica e dor à evacuação ${ }^{4}$.

A endometriose possui três apresentações diversas quanto ao aspecto clínico e de imagem: superficial, ovariana e profunda (EP). A EP é compreendida histologicamente como lesões que penetram mais que $5 \mathrm{~mm}$ no peritônio ${ }^{5}$. Já no exame clínico revela limitações para determinar a extensão das lesões endometrióticas profundas ${ }^{6,7}$. Dessa forma, busca-se a necessidade da utilização de outras formas diagnósticas como auxílio para estadiamento da doença. Durante muito tempo a videolaparoscopia foi o único método considerado adequado para este fim, restando para os métodos de imagem, principalmente ultrassom transvaginal e ressonância magnética, o papel de auxiliar na avaliação da endometriose ovariana $^{8}$.

Portanto, para o diagnóstico preciso da endometriose e a decisão sobre o tipo de tratamento a ser implantado, necessitase de uma boa anamnese, exame físico (principalmente toque vaginal), exames laboratoriais e de imagem $^{8}$. Logo, a ultrassonografia endovaginal tem sido um dos métodos de excelência no diagnóstico da endometriose profunda, sobretudo a intestinal, vesical e dos uterosacros ${ }^{9}$.

Segundo a Sociedade Brasileira de Endometriose e Ginecologia Mínimamente Invasiva (SBE), a doença vem se tornando nos últimos anos um problema de saúde publica, com morbidade e alto custo inquestionáveis. Conforme Nnoaham $^{10}$ mulheres com endometriose, confirmadas cirurgicamente, perdem $38 \%$ de sua capacidade de trabalho, representando um grande impacto socioeconômico, além da redução impactante na sua qualidade de vida.

Dessa forma, a suspeita da endometriose pela paciente é com vista no diagnóstico preciso, no qual contribui para elucidar fatores para um bom acompanhamento e na intervenção precoce, apontando indicativos importantes no que tange a qualidade de vida e sua influência no comprometimento da saúde.

Portanto a importância desta revisão é abordar o tema na qual é de extrema relevância para saúde da mulher e discutir as melhores formas diagnósticas atuais, em especial, a ultrassonografia, envolvidas na endometriose.

\section{Métodos}

Trata-se de um estudo de revisão de literatura com base nos principais artigos 
publicados sobre Endometriose nos últimos 10 anos. Os dados foram coletados através da consulta de revistas nacionais nos bancos de dados SCIELO, Medline e Pubmed. Foram utilizados os seguintes descritores: endometriose, diagnóstico, e acurácia da ultrassonografia.

\section{Revisão de literatura}

\section{Endometriose}

A endometriose é caracterizada pela presença de glândulas ou estroma endometrial fora do útero. A doença manifesta-se geralmente por dismenorreia, dor pélvica, infertilidade, dispareunia e alterações na urina e intestino durante 0 ciclo menstrual, indicando 0 seu caráter hormônio dependente. A dor pélvica se manifesta de várias maneiras, sendo dismenorreia a mais comum (79\%); a dispareunia está presente em $45 \%$ dos casos ${ }^{9}$.

A hipótese mais conhecida para a endometriose é a de Sampson em 1927. Segundo o autor ocorreria um refluxo de tecido endometrial através das trompas de falópio durante a menstruação, com consequente implantação e crescimento em tecidos adjacentes. Outras hipóteses propõe a possibilidade de metaplasia do epitélio celômico em tecido endometrial, disseminação linfática ou hematogênica do endométrio e ainda a diferenciação de células sanguíneas originárias da medula óssea em tecido endometrial ${ }^{9}$.

Outros estudos clínicos e experimentais demonstram que os estrogênios são necessários para que haja desenvolvimento da endometriose ${ }^{11}$, e, portanto ela está associada ao período reprodutivo da mulher ${ }^{12}$, exceto em casos isolados em que a doença ocorre em idades mais precoces ou avançadas. Moen et al..$^{13}$ estimam em $2 \%$ a incidência da endometriose em mulheres entre $40 \mathrm{e}$ 42 anos.

Para Kennedy et al. ${ }^{14}$ e Arruda et al. ${ }^{15}$, o diagnóstico definitivo da endometriose é cirúrgico. No entanto a suspeição e o diagnóstico clínico são fundamentais para 0 ginecologista desvendar esta enfermidade, envolvendo o atraso de anos notados entre o início dos sintomas até o reconhecimento da endometriose, evitando assim o tempo de sofrimentos destas mulheres. As manifestações clínicas da endometriose também são dor pélvica, dificuldade em engravidar e a presença de massa pélvica em mulheres na fase reprodutiva, de forma isolada ou em associações. Acompanhando essas condições é recomendável a investigação da endometriose. No entanto, estas manifestações por mais que sejam muito sugestivas, podem representar outras doenças e requerem 0 diagnóstico diferencial com outras condições: aderências, síndrome do intestino irritável, doença inflamatória pélvica, cistite, neoplasias e outras ${ }^{8}$.

Longos anos se passaram para que estabelecesse a abordagem da doença, constituindo-se, portanto, o conceito da infiltração das lesões e determinando-se assim a forma infiltrativa profunda da endometriose. Contudo, no final da década de 80, o desenvolvimento da cirurgia minimamente invasiva favoreceu o acesso cirúrgico menos traumático para a verificação da presença da doença e para a remoção direcionada das lesões. Logo, a evolução promoveu o direcionamento aos exames de imagem, em especial, o ultrassom transvaginal e pélvico e a ressonância nuclear magnética, e radiologistas especializados conseguiram identificar com alta acurácia as lesões profundas em seus diferentes sítios na pelve ${ }^{8}$.

\section{Ultrassonografia: acurácia diagnóstico da endometriose}

O exame clínico para a patologia apresenta limitações para estabelecer a extensão das lesões endometrióticas profundas, como já mencionado ${ }^{6,7}$. Portanto, houve a necessidade da busca 
de outras ferramentas para auxiliar no diagnóstico e estadiamento da doença. Durante algum tempo, a videolaparoscopia era a única forma considerada adequada para este objetivo, restando os métodos de imagem, em especial, a ultrassom transvaginal e ressonância magnética como medida fundamental no auxílio na avaliação da endometriose ovariana. A partir da década de 90, alguns pesquisadores publicaram, inicialmente, utilizando ultrassom transretal ou endoscópico e em seguida a ressonância magnética e ultrassom transvaginal, avaliando a acurácia desses métodos no diagnóstico da Endometriose Profunda $(E P)^{8}$. Atualmente o ultrassom transvaginal (USTV) e a RM magnética são os principais métodos na detecção e estadiamento da endometriose ${ }^{16,17}$. O USTV possui excelente sensibilidade e especificidade no diagnóstico do endometrioma de ovário, principalmente em lesões acima de $2 \mathrm{~cm}^{18}$. Guerriero et al. ${ }^{19}$, avaliaram 170 massas anexiais com USTV associado a Doppler colorido, em um estudo duplo cego, resultando em $97 \%$ de sensibilidade e especificidade de $90 \%$ e valor preditivo positivo e negativo de 95\% no diagnóstico do endometrioma ovariano.

O enema opaco e a colonoscopia apresentam baixa sensibilidade $e$, principalmente, especificidade para diagnóstico da EP intestinal, já que avaliam somente a superfície interna e o calibre da alça. Portanto, a não ser que haja lesão da mucosa, podem detectar somente sinais indiretos de endometriose. A tomografia computadorizada (TC), principalmente os novos equipamentos com múltiplos detectores, demonstram toda a espessura da parede, mas, ao contrário da ressonância magnética $(\mathrm{RM})$, não possui uma notável capacidade para diferenciar entre diversos tecidos de partes moles, dificultando a diferenciação e delimitação dos órgãos pélvicos e das lesões ${ }^{17}$.

Portanto, o método de escolha para a pesquisa de endometriose e estadiamento pré-operatório da endometriose profunda, na maioria dos casos é o exame de imagem, devendo ser realizado por profissional treinado e com um preparo intestinal simples, nos quais se incluem o laxante via oral na véspera e enema uma a duas horas antes do exame. Esse método do preparo intestinal visa a melhoria da acurácia do exame proporcionando melhor visualização do retossigmóide, dos anexos e da região retrocervical, na qual aumenta a sensibilidade do método para o diagnóstico dos focos de endometriose profunda. $O$ exame apresenta sensibilidade de $98 \%$ para identificação de endometriose, acometendo o retossigmoide, e de $95 \%$ para endometriose profunda na região retrocervical, com especificidade de $100 \mathrm{e}$ 98\% para essas duas localizações, respectivamente.

Segundo pesquisa com 1600 médicos do Estado de São Paulo, estes ressaltaram a ultrassonografia transvaginal como preferida como método diagnóstico da endometriose em $80.4 \%$ de $90 \%$. A ultrassonografia transvaginal é o ponto inicial de investigação da endometriose. Após o preparo intestinal possui a capacidade de rastreamento, diagnóstico e monitoramento das lesões intestinais que se apresentam como nódulos hipoecogênicos nos locais afetados, enfatizando como método de escolha para diferenciação entre endometriomas e cistos ovarianos ${ }^{9}$.

Gonçalves et al. ${ }^{20}$ demonstraram 194 pacientes utilizando a USGTV com preparo intestinal, cujo método diagnóstico na detecção de nódulos endometrióticos intestinais, resultando em sensibilidade de $97 \%$, uma especificidade de $100 \%$, VPP de $100 \%$ e VPN de $98 \%$. Já para a detecção de infiltração submucosa-mucosa a USGTV tem uma sensibilidade de $83 \%$, especificidade de $94 \%$, VPP de $77 \%$ e VPN de $96 \%$. Em outro estudo, Cruz et al. ${ }^{21}$ realizaram a USGTV e alcançaram 96\% de sensibilidade e $97 \%$ de especificidade no diagnóstico e mapeamento das lesões de endometriose profunda e do septo 
retovaginal, bexiga e intestino e sendo adequado na definição do tipo de cirurgia. A USGTV no reconhecimento da infiltração da endometriose intestinal seromuscular tem uma sensibilidade de $99 \%$, especificidade de $98 \%$, VPP de $98 \%$, VPN de $99 \%$ e acurácia de $99 \%$.

No que se refere à endometriose submucoso-mucosa, segundo Hudelist et al. ${ }^{22}$ possui uma sensibilidade de $96 \%$, especificidade de $62 \%$, VPP de $53 \%$, VPN de $97 \%$ e acurácia de $93,8 \%$. O estudo destaca ainda que, a USGTV é precisa na detecção seromuscular e menos útil na detecção da infiltração submucosamucosa. Gonçalves et al. ${ }^{20}$ em estudo com a USTV com preparo intestinal, obtiveram boa sensibilidade e especificidade para estabelecer a existência de mais de um nódulo de retossigmóide (81\%/99\%) e para as camadas comprometidas (100\%/100\% para infiltração até pelo menos a muscular própria e 83\%/94\% para infiltração da submucosa/ mucosa)

Portanto, evidenciou-se que o USTV é um método relevante para avaliar o tamanho das lesões intestinais, inclusive no eixo transversal, o que favorece estimar a circunferência da alça comprometida $^{20}$. Estudos recentes abordam que a ultrassom transabdominal associado ao transvaginal nos sítios intestinais é um método de primeira escolha para as pacientes com suspeita de endometriose. Alguns estudos científicos publicados na literatura demonstram o exame de USTV realizado sem preparo intestinal, porém, no nosso meio, predomina o exame com preparo intestinal prévio, pela maior facilidade na detecção do(s) nódulo(s), determinação das camadas e da distância da borda anal. O USTV também têm um papel fundamental no controle evolutivo do tratamento clínico e/ou cirúrgico ${ }^{7,20,23 .}$

\section{Conclusão}

À partir da revisão de literatura foi possível concluir que a USTV demonstrou obter uma excelente sensibilidade e acurácia como exame de rastreamento, diagnóstico e monitoramento da endometriose, apresentando qualidade na resolução da estratificação, na avaliação do grau de infiltração tecidual, além de ser eficiente no acompanhamento e tratamento clínico medicamentoso e cirúrgico.

\section{Referências}

1. Ezer SS, Tarim E, Parlakgumus HA. Antenatal diagnosis of sacrococcygeal teratoma-two different case reports. Ginekologia polska. 2009;80(6).

2. Wilson RD, Hedrick H, Flake AW, Johnson MP, Bebbington MW, Mann S, et al. Sacrococcygeal teratomas: prenatal surveillance, growth and pregnancy outcome. Fetal diagnosis and therapy. 2009;25(1):15-20.

3. Duleba AJ. Diagnosis of endometriosis. Obstetrics and gynecology clinics of North America. 1997;24(2):331-46.

4. Mowers EL, Lim CS, Skinner B, Mahnert N, Kamdar N, Morgan DM, et al. Prevalence of endometriosis during abdominal or laparoscopic hysterectomy for chronic pelvic pain. Obstetrics \& Gynecology. 2016;127(6):1045-53.

5. Koninckx PR, Meuleman C, Demeyere S, Lesaffre E, Cornillie FJ. Suggestive evidence that pelvic endometriosis is a progressive disease, whereas deeply infiltrating endometriosis is associated with pelvic pain. Fertility and sterility. 1991;55(4):759-65.

6. Chapron C, Dubuisson J-B, Pansini V, Vieira M, Fauconnier A, Barakat $\mathrm{H}$, et al. Routine clinical examination is not sufficient for diagnosing and locating deeply infiltrating endometriosis. The Journal of the American Association of Gynecologic Laparoscopists. 2002;9(2):115-9.

7. Abrao MS, Gonçalves MOdC, Dias Jr JA, Podgaec S, Chamie LP, Blasbalg R. Comparison between clinical examination, transvaginal sonography and magnetic resonance imaging for the diagnosis of deep endometriosis. Human Reproduction. 2007;22(12):3092-7. 
8. Podgaec S. Endometriose: Coleção Febrasgo: Elsevier Brasil; 2015.

9. De amaral, Teixeira A A, Falone VE, Carolina I D, Moraes W N, Filho da, Do Amaral W N. A ultrassonografia transvaginal no diagnóstico da endometriose profunda. março de $201518^{\text {a }}$ edição. 2015:34.

10.Nnoaham KE, Hummelshoj L, Webster P, d'Hooghe $T$, de Cicco Nardone $F$, de Cicco Nardone $\mathrm{C}$, et al. Impact of endometriosis on quality of life and work productivity: a multicenter study across ten countries. Fertility and sterility. 2011;96(2):366-73. e8.

11.Vercellini P, Trespidi L, De Giorgi O, Cortesi I, Parazzini F, Crosignani PG. Endometriosis and pelvic pain: relation to disease stage and localization. Fertility and sterility. 1996;65(2):299-304.

12. Eskenazi B, Warner ML. Epidemiology of endometriosis. Obstetrics and gynecology clinics of North America. 1997;24(2):235-58.

13. Moen $\mathrm{MH}$, Schei B. Epidemiology of endometriosis in a Norwegian county. Acta obstetricia et gynecologica Scandinavica. 1997;76(6):559-62.

14. Kennedy S, Bergqvist A, Chapron C, D'hooghe T, Dunselman G, Greb R, et al. ESHRE guideline for the diagnosis and treatment of endometriosis. Human reproduction. 2005;20(10):2698-704

15.Arruda M, Petta C, Abrao M, Benetti-Pinto C. Time elapsed from onset of symptoms to diagnosis of endometriosis in a cohort study of Brazilian women. Human Reproduction. 2003;18(4):756-9.

16. Bazot M, Darai E, Hourani R, Thomassin I, Cortez A, Uzan S, et al. Deep pelvic endometriosis: MR imaging for diagnosis and prediction of extension of disease. Radiology. 2004;232(2):379-89.

17.Abrão MS, Neme RM, Averbach M, Petta CA, Aldrighi JM. Rectal endoscopic ultrasound with a radial probe in the assessment of rectovaginal endometriosis. The Journal of the American Association of Gynecologic Laparoscopists. 2004;11(1):50-4.

18. Moore J, Copley S, Morris J, Lindsell D, Golding S, Kennedy S. A systematic review of the accuracy of ultrasound in the diagnosis of endometriosis. Ultrasound in Obstetrics and Gynecology: The Official Journal of the International Society of Ultrasound in Obstetrics and Gynecology. 2002;20(6):630-4.

19.Guerriero S, Ajossa S, Risalvato A, Lai M, Mais $V$, Angiolucci $M$, et al. Diagnosis of adnexal malignancies by using color Doppler energy imaging as a secondary test in persistent masses. Ultrasound in Obstetrics and Gynecology: The Official Journal of the International Society of Ultrasound in Obstetrics and Gynecology. 1998;11(4):277-82.

20.Goncalves MOdC, Podgaec S, Dias Jr JA, Gonzalez M, Abrao MS. Transvaginal ultrasonography with bowel preparation is able to predict the number of lesions and rectosigmoid layers affected in cases of deep endometriosis, defining surgical strategy. Human reproduction. 2009;25(3):665-71.

21.Cruz R, Amaral W, AmaraL Filho W. Diagnóstico ultrassonográfico da endometriose pelvica. RBUS. 2009;10:21-3.

22. Hudelist G, Tuttlies F, Rauter G, Pucher S, Keckstein J. Can transvaginal sonography predict infiltration depth in patients with deep infiltrating endometriosis of the rectum? Human reproduction. 2009;24(5):1012-7.

23.Piketty $M$, Chopin N, Dousset B, MillischerBellaische A-E, Roseau $G$, Leconte $M$, et al. Preoperative work-up for patients with deeply infiltrating endometriosis: transvaginal ultrasonography must definitely be the first-line imaging examination. Human Reproduction. 2008;24(3):602-7. 